\title{
Unsteady Aerodynamic Response of Oscillating Contra-Rotating Annular Cascades Part I: Description of Model and Mathematical Formulations
}

\author{
By Masanobu NAMBA and Ryohei NISHINO \\ Department of Aerospace Systems Engineering, Sojo University, Kumamoto, Japan \\ (Received January 24th, 2006)
}

\begin{abstract}
The purpose of this paper is to study the effect of neighboring blade rows on the unsteady aerodynamic response of oscillating cascade blades on the basis of a genuine three-dimensional model. To this end, mathematical formulations based on the lifting surface theory are developed for a pair of contra-rotating annular cascades of oscillating blades. The mechanism of frequency scattering of blade loadings and mode scattering of acoustic waves resulting from interaction between the blade rows in relative rotational motions is mathematically explained. Simultaneous integral equations for all frequency components of blade loadings are derived from the flow tangency condition on the blade surfaces of both blade rows. The validity of the computation codes is verified.
\end{abstract}

Key Words: Cascade Flutter, Multiple Blade Rows, Counter Rotation, Annular Cascade, Rotor-Stator Interaction, Unsteady Aerodynamics, Lifting Surface Theory

\section{Nomenclature}

$a_{j}(r, z)$ : displacement amplitude of a blade of rotor $j$ normal to the blade surface

$C_{a}:=\left(C_{a 1}+C_{a 2}\right) / 2$

$C_{a j}$ : axial chord length of blades of rotor $j$

$C_{F j}$ : generalized force coefficient of rotor $j$ defined by Eq. (19)

$C_{L j,(\mu)}(r)$ : unsteady local lift coefficient of harmonic number $\mu$ of rotor $j$ defined by Eq. (17)

$C_{M j,(\mu)}(r)$ : unsteady local moment coefficient of harmonic number $\mu$ of rotor $j$ defined by Eq. (18)

$G$ : axial distance between centers of rotor 1 and rotor 2

$h$ : hub/tip ratio

$\boldsymbol{K}_{q}:$ velocity kernel function

$\boldsymbol{K}_{q}{ }^{(v, \ell)}$ : modal component of $\boldsymbol{K}_{q}$ of circumferential harmonic number $v$ and radial order $\ell$

$k_{\ell}^{n}$ : radial eigenvalue

$L$ : maximum radial order retained in numerical calculation

$\ell$ : radial order of acoustic duct mode

$M_{a}$ : axial Mach number

$N_{B j}$ : number of blades of rotor $j$

$n_{\mu, \nu}$ : circumferential wave number of acoustic duct mode of harmonic parameters $\mu$ and $v$

$\boldsymbol{q}$ : disturbance fluid flow velocity

$q_{\perp j}$ : component of $\boldsymbol{q}$ normal to blade surface of rotor $j$

$R_{\ell}^{(n)}(r)$ : radial eigenfunction of radial order $\ell$ and circumferential wave number $n$

$r_{T}^{*}$ : radius of the cylindrical duct (dimensional value)

(C) 2006 The Japan Society for Aeronautical and Space Sciences $(r, \theta, z)$ : cylindrical coordinate system fixed to duct

$\left(r, \theta_{j}, z_{j}\right)$ : cylindrical coordinate system fixed to rotor $j$

$t$ : time coordinate

$W_{a}{ }^{*}$ : axial flow velocity of undisturbed fluid (dimensional value)

$\Delta p_{j,(\mu)}(r, z)$ : unsteady blade loading of harmonic number $\mu$ of rotor $j$

$\delta_{i j}$ : Kronecker delta

$\eta_{j}:=\theta_{j}-\Omega_{j} z_{j}:$ helical coordinate along undisturbed streamline relative to rotor $j$

$\mu_{\max }:$ maximum circumferential mode parameter

$\rho_{0}{ }^{*}$ : undisturbed fluid density (dimensional value)

$\Omega_{j}$ : rotational velocity of rotor $j$ normalized by $W_{a}{ }^{*} / r_{T}{ }^{*}$

$\omega$ : reduced frequency of blade vibration normalized by $W_{a}{ }^{*} / r_{T}{ }^{*}$

$\omega_{j \mu}$ : reduced frequency of disturbance flow component of harmonic number $\mu$ for $\left(r, \theta_{j}, z_{j}\right)$ system

$\omega_{\mu, v}$ : reduced frequency of disturbance flow of harmonic component $(\mu, v)$ for duct fixed coordinate system

$\sigma$ : interblade phase parameter of blade vibration

$\sigma_{j \mu}$ : interblade phase parameter of fluctuating disturbance flow component of harmonic number $\mu$ for $\left(r, \theta_{j}, z_{j}\right)$ system

$\Im[$ ]: imaginary part of complex number

\section{Introduction}

Cascade flutter is characterized by the aerodynamic interaction between oscillating blades in a blade row. Its importance can be understood from the fact that the unsteady aerodynamic force on oscillating blades is heavily dependent on 
the inter-blade phase angle of oscillation. From this standpoint, neighboring blade rows (e.g., a neighboring rotor or stator or contra-rotating fan cascade) also have a considerable influence on the unsteady aerodynamic force because blade rows are closely placed in actual turbomachines.

There are a few published papers available dealing with the influence of neighboring blade rows on the unsteady aerodynamic response of oscillating blades. The theoretical study based on the semi-actuator disk model by Tanida ${ }^{1)}$ and the experimental and theoretical studies based on the linear cascade model by Kobayashi et al. ${ }^{2,3)}$ should be cited as the earliest works. Their works express a minor importance of the multistage coupling for cascade flutter, and this may be one of the reasons why further studies have not been conducted. It should be noted, however, that their theories assume incompressible flows and therefore cannot deal with aeroacoustic interaction between blade rows via cut-on acoustic duct modes.

There is also a Russian paper by Butenko and Osipov, ${ }^{4)}$ which developed a theory for subsonic linear cascades in relative motion.

Extending the study of cascade flutter to multistage cascades is recently drawing renewed interest from turbomachinery aerodynamicists. Hall and Silkowski ${ }^{5)}$ presented an analysis based on two-dimensional subsonic multiple blade rows, and Namba et al. ${ }^{6}$ developed a three-dimensional lifting surface theory for oscillating subsonic contra-rotating annular cascades. Those studies of subsonic flows indicate that the influence of aeroacoustic coupling among blade rows on the aerodynamic damping force is significant, in particular when the main acoustic duct mode generated from the oscillating blade row is of cut-on state.

The lifting surface theory has further been extended ${ }^{7)}$ so that it can deal with the combination of supersonic and subsonic cascades, for instance, a supersonic rotor cascade and a subsonic stator cascade. Hall et al. ${ }^{8)}$ developed a three-dimensional Euler solver for computing unsteady flows in vibrating multistage cascades. In their paper, a comparison is made of a model problem between the computations by their Euler solver and the author's lifting surface theory, and excellent agreement is demonstrated.

This paper (comosed of Part I and Part II) gives the details of an analytical study on contra-rotating annular cascades with oscillating blades. One of the aims of the study is to investigate the effect of the presence of neighboring cascades on cascade flutter. A contra-rotating ducted fan itself is of special interest as one of the possible configurations of ultra-high bypass engines. The present model, of course, can be reduced to a rotor-stator model by taking the rotational speed of one of the rotors as zero. In this case, however, the stagger angle of the stator inevitably becomes zero because the present linearized analysis can only deal with nearly uniform axial flows. On the other hand, in the case of contra-rotating cascades, the stagger angles which are dependent on the rotational speeds can be arbitrarily specified in the scope of the linearized analysis.

Owing to the rapid improvement of computer perform- ance and computational techniques, CFD is becoming a practical tool in the theoretical studies of cascade aerodynamics. However, CFD is still too expensive for use in the study of the present multi-cascade model because it is three-dimensional, and above all, it involves the phenomena of multiple frequencies and multiple inter-blade phase angles.

In Part I of this paper, mathematical formulations for predicting unsteady loading on blades are outlined. The analytical method used in this paper is an extension of one of the authors' unsteady linearized lifting surface theories for a rotating annular cascade ${ }^{9-12)}$ to the model of a pair of contrarotating cascades. Recently, the validity of the original code has been confirmed by comparing the authors' data with those computed by Schulten's code, ${ }^{13)}$ both of which were submitted to the Third Computational Aeroacoustics Workshop on Benchmark Problems (November 1999, Ohio Aerospace Institute) as analytical solutions of Category 4 - Fan Stator with Harmonic Excitation by Rotor Wake. ${ }^{14)}$

As mentioned before, the validity of the present program to compute the unsteady aerodynamic response of an scillating blade row under the influence of a neighboring blade row in relative rotational motion has also been proved by comparison with the results of a CFD analysis. ${ }^{8}$

The applicability of the present linearized theory is restricted to the condition of small perturbation of a uniform axial flow. It is, however, a useful tool to investigate the general features of the influence of the neighboring blade row on cascade flutter because it can provide desired information with very short computation time on a current personal computer.

\section{Outline of the Analytical Method}

\subsection{Model description}

We consider a pair of annular cascades in an annular duct of infinite axial extent with the outer duct radius $r_{T}{ }^{*}$ and the boss ratio $h$ as shown in Fig. 1. The undisturbed flow is a uniform axial flow of axial velocity $W_{a}{ }^{*}$, static pressure $p_{0}{ }^{*}$ and fluid density $\rho_{0}{ }^{*}$. In the following, unstarred symbols denote dimensionless quantities, where lengths, velocities, pressures and times are scaled with respect to $r_{T}{ }^{*}, W_{a}{ }^{*}$, $\rho_{0}{ }^{*} W_{a}{ }^{* 2}$ and $r_{T}{ }^{*} / W_{a}{ }^{*}$, respectively. Let subscripts 1 and 2 denote the upstream rotor 1 and the downstream rotor 2 , respectively. Further the following notations are used: $N_{B 1}$
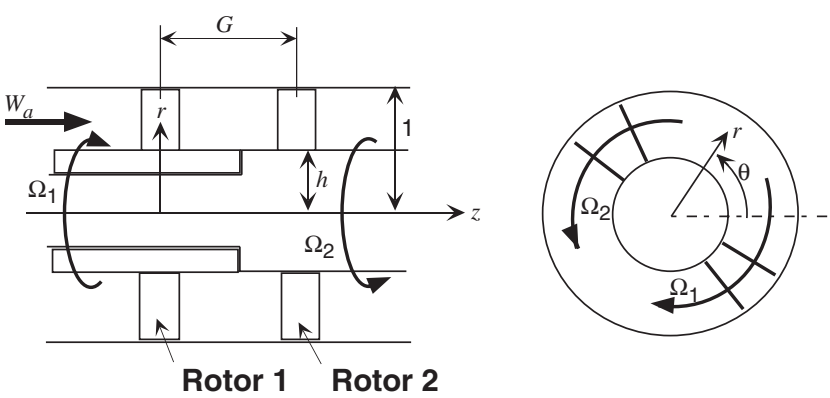

Fig. 1. Contra-rotating annular cascades 
and $N_{B 2}$, numbers of blades; $\Omega_{1}=\Omega_{1}{ }^{*} r_{T}{ }^{*} / W_{a}{ }^{*}(\geq 0$, clockwise looking from a downstream station) and $\Omega_{2}(\leq 0$, anticlockwise), rotational angular velocities of the rotors; $C_{a 1}$ and $C_{a 2}$, axial chord length assumed constant along the span; and $(r, \theta, z),\left(r, \theta_{1}, z_{1}\right)$ and $\left(r, \theta_{2}, z_{2}\right)$, cylindrical coordinates fixed to the duct, rotor 1 and rotor 2 , respectively. The angle coordinates and axial coordinates of different frames are related to each other by

$$
\theta=\theta_{1}-\Omega_{1} t=\theta_{2}-\Omega_{2} t, \quad z=z_{1}=z_{2}+G .
$$

Here, $t$ denotes the time coordinate and $G$ denotes the axial distance between the centers of rotor 1 and rotor 2 .

We assume that the steady blade loading is zero (i.e., time mean flow is uniform), and that the unsteady disturbances induced by blade vibrations are small. Therefore, the unsteady flow is governed by linearized equations.

\subsection{Multiplication mechanism of frequency and cir- cumferential mode}

Assume that all blades of rotor 1 are vibrating with a single frequency $\omega$ and an inter-blade phase angle $2 \pi \sigma / N_{B 1}$, so that the displacement normal to the blade chord of the $m$-th blade is given by

$$
a_{1}(r, z) e^{i \omega t+i 2 \pi \sigma m / N_{B 1}}: m=0,1, \ldots, N_{B 1}-1 .
$$

Here, $\sigma$ is an integer between $-N_{B 1} / 2$ and $N_{B 1} / 2$. This blade motion generates disturbances of time dependence and circumferential modes described as

$$
\begin{aligned}
& \sum_{\mu=-\infty}^{\infty} \boldsymbol{Q}_{\mu}(r, z) e^{i \omega t+i\left(\mu N_{B 1}+\sigma\right) \theta_{1}} \\
& =\sum_{\mu=-\infty}^{\infty} \boldsymbol{Q}_{\mu}(r, z) e^{i \omega_{2 \mu} t+i \sigma_{2 \mu} \theta_{2}}
\end{aligned}
$$

where

$$
\begin{gathered}
\omega_{2 \mu}=\omega+\left(\mu N_{B 1}+\sigma\right) \Omega_{12}, \quad \sigma_{2 \mu}=\mu N_{B 1}+\sigma, \\
\Omega_{12}=\Omega_{1}-\Omega_{2} .
\end{gathered}
$$

Note that the left-hand side of Eq. (3) describes disturbances viewed in the frame of reference fixed to rotor 1 , which is converted into the right-hand side by Eq. (1) as viewed in the frame of reference fixed to rotor 2. This implies that the blades of rotor 2 sense the disturbances as those composed of multiple frequencies $\omega_{2 \mu}=\omega+\left(\mu N_{B 1}+\sigma\right) \Omega_{12}$ and multiple inter-blade phase angles $2 \pi \sigma_{2 \mu} / N_{B 2}=$ $2 \pi\left(\mu N_{B 1}+\sigma\right) / N_{B 2}:(\mu=0, \pm 1, \pm 2, \ldots)$.

On the other hand, the interaction of these disturbances with rotor 2 blades regenerates (reflects back) the disturbances of time dependence and circumferential modes described as

$$
\begin{aligned}
& \sum_{\nu=-\infty}^{\infty} \sum_{\mu=-\infty}^{\infty} \boldsymbol{Q}_{\mu \nu}{ }^{\prime}(r, z) e^{i \omega_{2 \mu} t+i\left(\nu N_{B 2}+\sigma_{2 \mu}\right) \theta_{2}} \\
& =\sum_{\nu=-\infty}^{\infty} \sum_{\mu=-\infty}^{\infty} \boldsymbol{Q}_{\mu \nu}{ }^{\prime}(r, z) e^{i \omega_{1 \nu} t+i\left(\mu N_{B 1}+\sigma_{1 v}\right) \theta_{1}},
\end{aligned}
$$

where

$$
\omega_{1 v}=\omega-v N_{B 2} \Omega_{12}, \quad \sigma_{1 v}=v N_{B 2}+\sigma .
$$

Again, conversion from the left-hand side into the righthand side of Eq. (6) as viewed in the frame of reference fixed to rotor 1 is made using Eq. (1).

Equation (6) indicates that the unsteady blade loading (pressure difference between the upper and lower surfaces of the blades) on the $m$-th blade of each rotor should be described as summations of multiple frequency components:

$$
\begin{aligned}
& \sum_{\nu=-\infty}^{\infty} \Delta p_{1,(v)}\left(r, z_{1}\right) e^{i \omega_{1 \nu} t+i 2 \pi \sigma_{1 \nu} m / N_{B 1}}, \\
& \sum_{\mu=-\infty}^{\infty} \Delta p_{2,(\mu)}\left(r, z_{2}\right) e^{i \omega_{2 \mu} t+i 2 \pi \sigma_{2 \mu} m / N_{B 2}} .
\end{aligned}
$$

Here, subscripts 1 and 2 imply the loading on rotor 1 blades and the loading on rotor 2 blades, respectively. It is worth emphasizing that all frequency components are aerodynamically coupled with each other and cannot be determined independently.

\subsection{Solution philosophy}

The fundamental concept of the lifting surface theory is to regard the unsteady blade loadings as the disturbance sources and to express the disturbance flow field as the summation of disturbances induced by the unsteady blade loadings of both rotors. In the case of the vibration of rotor 1 blades as described above, the disturbance velocity $\boldsymbol{q}$ is expressed in the form of

$$
\begin{aligned}
\boldsymbol{q}= & \sum_{\nu=-\infty}^{\infty} e^{i \omega_{1 v} t} \int_{h}^{1} \int_{-C_{a 1} / 2}^{C_{a 1} / 2} \Delta p_{1,(v)}(\rho, \zeta) \\
& \times \boldsymbol{K}_{q}\left(r, \eta_{1}, z_{1}-\zeta \mid \rho ; N_{B 1}, \Omega_{1}, \omega_{1 v}, \sigma_{1 v}\right) \mathrm{d} \zeta \mathrm{d} \rho \\
& +\sum_{\mu=-\infty}^{\infty} e^{i \omega_{2 \mu} t} \int_{h}^{1} \int_{-C_{a 2} / 2}^{C_{a 2} / 2} \Delta p_{2,(\mu)}(\rho, \zeta) \\
& \times \boldsymbol{K}_{q}\left(r, \eta_{2}, z_{2}-\zeta \mid \rho ; N_{B 2}, \Omega_{2}, \omega_{2 \mu}, \sigma_{2 \mu}\right) \mathrm{d} \zeta \mathrm{d} \rho
\end{aligned}
$$

where $\eta_{1}$ and $\eta_{2}$ are helical coordinates defined by

$$
\eta_{1}=\theta_{1}-\Omega_{1} z_{1}, \quad \eta_{2}=\theta_{2}-\Omega_{2} z_{2} .
$$

Note that $\eta_{j}=$ constant and $r=$ constant give an undisturbed streamline in the frame of refefence fixed to rotor $j$, and the $m$-th blade of rotor $j$ is placed on the surface $\eta_{j}=2 \pi m / N_{B j}$.

The kernel function $\boldsymbol{K}_{q}\left(r, \eta, z-\zeta \mid \rho ; N_{B}, \Omega, \omega, \sigma\right)$ denotes the disturbance velocity induced by an annular row of pressure dipoles, which are placed at $\eta=2 \pi m / N_{B}$ ( $\left.m=0,1, \ldots, N_{B}-1\right), r=\rho, z=\zeta$ and rotating at a dimensionless angular speed $\Omega$ with respect to the duct-fixed coordinated system. The axes of the dipoles are normal to the helical surfaces of $\eta=2 \pi m / N_{B} ;\left(m=0,1, \ldots, N_{B}\right)$. The strength of the dipoles is fluctuating with unit amplitude at a reduced frequency $\omega$ and a phase difference $2 \pi \sigma / N_{B}$ between one dipole and the next. The mathematical expression of the kernel function is exactly same as that given for a single cascade model. ${ }^{9-12)}$ It should be noted that it involves not only acoustic disturbances but also vortical disturbances 
convected downstream from the dipole points. This is further decomposed into circumferential modes and radial modes in the form of

$$
\begin{aligned}
\boldsymbol{K}_{q}(r, & \left.\eta_{j}, z_{j}-\zeta \mid \rho ; N_{B j}, \Omega_{j}, \omega_{j \mu}, \sigma_{j \mu}\right) \\
= & \sum_{\nu=-\infty}^{\infty} e^{i\left(\nu N_{B j}+\sigma_{j \mu}\right) \eta_{j}} \sum_{\ell=0}^{\infty} R_{\ell}^{(n)}(r) \\
& \times \boldsymbol{K}_{q}^{(\nu, \ell)}\left(z_{j}-\zeta \mid \rho ; N_{B j}, \Omega_{j}, \omega_{j \mu}, \sigma_{j \mu}\right) .
\end{aligned}
$$

Here, $n=v N_{B j}+\sigma_{j \mu}=v N_{B j}+\mu N_{B k}+\sigma$ is a circumferential wave number and $R_{\ell}^{(n)}(r)$ denotes a radial eigenfunction of order $\ell$ as used in the previous papers dealing with a single blade row model. ${ }^{9,10)}$

Equation (10) describes the disturbance flow velocity field in terms of the unsteady blade loadings as the disturbance sources. It should be noted, however, that in the present problem, the blade loadings are not prescribed but should be determined so that the disturbance velocity given by Eq. (10) satisfies the flow tangency condition on the oscil- lating or stationary blade surfaces of both rotors. It is further worth emphasizing that the true disturbance source is the oscillating motion of the blades of rotor 1 , and the expression (10) with the blade loading functions determined in this way automatically includes the phenomena of reflection and transmission of disturbances at the rotors.

The flow tangency condition at the blade surfaces can be expressed by

$$
\begin{gathered}
{\left[q_{\perp 1}\right]_{\eta_{1}=0}=\left(i \omega a_{1}\left(r, z_{1}\right)+\frac{\partial a_{1}\left(r, z_{1}\right)}{\partial z_{1}}\right) e^{i \omega t}} \\
:-\frac{C_{a 1}}{2} \leq z_{1} \leq \frac{C_{a 1}}{2}, \\
{\left[q_{\perp 2}\right]_{\eta_{2}=0}=0:-\frac{C_{a 2}}{2} \leq z_{2} \leq \frac{C_{a 2}}{2}}
\end{gathered}
$$

where $q_{\perp j}$ denotes the disturbance velocity component normal to the blade surfaces of rotor $j$. Equations (13) and (14) can be rewritten into a set of simultaneous integral equations for the unsteady blade loading functions as follows:

$$
\begin{aligned}
& \int_{h}^{1} \int_{-C_{a 1} / 2}^{C_{a 1} / 2} \Delta p_{1,(v)}(\rho, \zeta) K_{q \perp 1}\left(r, 0, z_{1}-\zeta \mid \rho ; N_{B 1}, \Omega_{1}, \omega_{1 \nu}, \sigma_{1 \nu}\right) \mathrm{d} \zeta \mathrm{d} \rho \\
& \quad+\sum_{\mu=-\infty}^{\infty} e^{i\left(\mu N_{B 1}+\sigma_{1 v}\right)\left(\Omega_{12} z_{1}+\Omega_{2} G\right)} \int_{h}^{1} \int_{-C_{a 2} / 2}^{C_{a 2} / 2} \Delta p_{2,(\mu)}(\rho, \zeta) \sum_{\ell=0}^{\infty} R_{\ell}^{(n)}(r) K_{q \perp 1}^{(\nu, \ell)}\left(z_{1}-G-\zeta \mid \rho ; N_{B 2}, \Omega_{2}, \omega_{2 \mu}, \sigma_{2 \mu}\right) \mathrm{d} \zeta \mathrm{d} \rho \\
& \quad=\left(i \omega a_{1}\left(r, z_{1}\right)+\frac{\partial a_{1}\left(r, z_{1}\right)}{\partial z_{1}}\right) \delta_{\nu 0}:-\frac{C_{a 1}}{2} \leq z_{1} \leq \frac{C_{a 1}}{2}, \\
& \int_{h}^{1} \int_{-C_{a 2} / 2}^{C_{a 2} / 2} \Delta p_{2,(\nu)}(\rho, \zeta) K_{q \perp 2}\left(r, 0, z_{2}-\zeta \mid \rho ; N_{B 2}, \Omega_{2}, \omega_{2 v}, \sigma_{2 v}\right) \mathrm{d} \zeta \mathrm{d} \rho \\
& \quad+\sum_{\mu=-\infty}^{\infty} e^{-i\left(\mu N_{B 2}+\sigma_{2 v}\right)\left(\Omega_{12} z_{2}+\Omega_{1} G\right)} \int_{h}^{1} \int_{-C_{a 1} / 2}^{C_{a 1} / 2} \Delta p_{1,(\mu)}(\rho, \zeta) \sum_{\ell=0}^{\infty} R_{\ell}^{(n)}(r) K_{q \perp 2}^{(v, \ell)}\left(z_{2}+G-\zeta \mid \rho ; N_{B 1}, \Omega_{1}, \omega_{1 \mu}, \sigma_{1 \mu}\right) \mathrm{d} \zeta \mathrm{d} \rho \\
& \quad=0:-\frac{C_{a 2}}{2} \leq z_{2} \leq \frac{C_{a 2}}{2} . \\
& v=0, \pm 1, \pm 2, \ldots
\end{aligned}
$$

Here, $\delta_{\nu 0}$ denotes Kronecker delta, and $K_{q \perp j}$ and $K_{q \perp j}^{(\nu, \ell)}$ denote components of $\boldsymbol{K}_{q j}$ and $\boldsymbol{K}_{q j}^{(v, \ell)}$ normal to the blade surfaces of rotor $j$, respectively.

To numerically solve Eqs. (15) and (16), the infinite series with respect to $\mu$ is truncated into a finite series up to say, $|\mu|=\mu_{\max }$. The truncation is accompanied by truncation of the number of equations up to $v= \pm \mu_{\max }$, too. This implies omitting the frequency components of the loading functions $\Delta p_{1,(v)}(\rho, \zeta)$ and $\Delta p_{2,(v)}(\rho, \zeta)$ of parameter $v$ higher than $\mu_{\text {max }}$. The validity of the approximation from the standpoint of evaluation of aerodynamic work on oscillating blades will be examined in Part II.

Truncating the infinite series into a finite series, one can numerically solve the equations by a standard method. In this paper, the blade loading functions, $\Delta p_{1,(v)}(\rho, \zeta)$ and $\Delta p_{2,(v)}(\rho, \zeta)$, are expressed in terms of double mode function series, and Eqs. (15) and (16) are converted into algebraic equations for the coefficients of the mode function series as used by $\mathrm{Li}$ et al. ${ }^{11,12)}$
In the case of blade the vibration of rotor 2 , similar formulations can be made, but the details are omitted to save space.

\subsection{Unsteady aerodynamic force and work}

As Eqs. (8) and (9) show, the unsteady loading on a blade is composed of an infinite number of frequency components. We define the frequency components of the local unsteady lift coefficient $C_{L j,(v)}(r)$ and the unsteady local moment coefficient about the mid-chord $C_{M j,(v)}(r)$ of rotor $j(j=1$ or 2) by

$$
\begin{aligned}
C_{L j,(v)}(r) & =\frac{1}{A C_{a j}} \int_{-C_{a j} / 2}^{C_{a j} / 2} \Delta p_{j,(v)}\left(r, z_{j}\right) \mathrm{d} z_{j}, \\
C_{M j,(v)}(r) & =\frac{1}{A C_{a j}^{2}} \int_{-C_{a j} / 2}^{C_{a j} / 2} \Delta p_{j,(v)}\left(r, z_{j}\right) z_{j} \mathrm{~d} z_{j} .
\end{aligned}
$$

Here, $A$ denotes the displacement amplitude of the blade oscillation.

In order to evaluate the aerodynamic work on oscillating blades, we define generalized force coefficients by 
$C_{F j}=\frac{1}{A^{2}} \pi \int_{h}^{1} \sqrt{1+\Omega_{j}^{2} r^{2}} \int_{-C_{a j} / 2}^{C_{a j} / 2} \Delta p_{j,(0)}\left(r, z_{j}\right) \overline{a_{j}\left(r, z_{j}\right)} \mathrm{d} z_{j} \mathrm{~d} r$,

where an overlined symbol denotes the complex conjugate. Here $j$ denotes the rotor number ( 1 or 2 ) blades of which are oscillating. For example, consider the case $j=1$. Then the unsteady loading on an oscillating blade is composed of an infinite number of frequency components $\Delta p_{1,(v)}\left(r, z_{1}\right) e^{i\left(\omega-v N_{B 2} \Omega_{12}\right) t}: v=0, \pm 1, \pm, 2, \ldots$ It should be noted, however, that only the component of $v=0$ (i.e., the component of the frequency $\omega_{1 v}=\omega-v N_{B 2} \Omega_{12}$ that is same as the blade motion frequency $\omega$ ) contributes to the aerodynamic work per cycle. In fact, the imaginary part of the generalized force coefficient corresponds to dimensionless aerodynamic work per cycle on an oscillating blade. Therefore, we can define the aerodynamic work coefficient $W_{j}$ by

$$
W_{j}=\Im\left[C_{F j}\right] .
$$

\subsection{Acoustic modes and frequencies}

In the present problem, disturbed flow quantities are composed of multiple frequencies. The frequencies $\omega_{v, \mu}$ viewed in the frame of reference fixed to the duct (i.e., $(r, \theta, z)$ system) and corresponding circumferential wave numbers $n_{\mu, v}$ are given in Table 1.

Hereafter, we denote the acoustic mode of $\left(n_{\mu, \nu}, \ell\right)$ by $(\mu, v ; \ell)$, where $\ell$ denotes the radial order. A mode $(\mu, v ; \ell)$ is cut-on if

$$
\left(k_{\ell}^{\left(n_{\mu, v}\right)}\right)^{2}-\omega_{\mu, \nu}{ }^{2} M_{a}^{2} /\left(1-M_{a}^{2}\right)<0 .
$$

Here, $k_{\ell}^{\left(n_{\mu . v}\right)}$ denotes the radial eigenvalue for the duct mode of circumferential wave number $n_{\mu, \nu}$ and the radial node number $\ell .^{9,10)}$

Under this notation, we can state that oscillating blades directly generate $(\mu, 0 ; \ell)$ modes. We call those modes primary modes. On the other hand, the modes of $v \neq 0$ are caused by the interaction between blade rows, and we call them secondary modes. If all of the primary modes are cut-off and if the rotors are remotely separated, the influence of the neighboring blade row will be weak. It should be noted, however, that there exist vortical disturbances which are shed from blades and convected without decaying. Therefore, in the case of the vibration of rotor 1, the vortical disturbances from rotor 1 always exert a finite influence on rotor 2 even if all primary acoustic duct modes $(\mu, 0 ; \ell)$ are cut-off, and no matter how large the rotor-to-rotor distance $G$ may be. Further, any of the modes of $v \neq 0$ resulting from the interaction can be of cut-on state, giving backward reaction to rotor 1.

Table 1. Frequencies and circumferential wave numbers.

\begin{tabular}{ccc}
\hline Case & Frequency $\omega_{\mu, \nu}$ & Wave number $n_{\mu, \nu}$ \\
\hline Vibration of rotor 1 & $\omega+\mu N_{B 1} \Omega_{1}+v N_{B 2} \Omega_{2}+\sigma \Omega_{1}$ & $\mu N_{B 1}+v N_{B 2}+\sigma$ \\
Vibration of rotor 2 & $\omega+\mu N_{B 2} \Omega_{2}+v N_{B 1} \Omega_{1}+\sigma \Omega_{2}$ & $\mu N_{B 2}+v N_{B 1}+\sigma$ \\
\hline
\end{tabular}

\section{Code Validation}

The mathematical formulations of the unsteady lifting surface theory for the present model are not simple, and the computation program to numerically solve the problem inevitably becomes complicated. To validate the present code, comparison with the existing results for a two-dimensional model was conducted by specifying a very narrow annular model: $h=0.99, M_{a}=0.35, \Omega_{1}=1.732, \Omega_{2}=$ $0.0, N_{B 1}=38, N_{B 2}=50, C_{a 1}=0.11023, C_{a 2}=0.12566$, $\omega C_{a 1}=1.0, \quad G=3.804\left(C_{a 1}+C_{a 2}\right) / 2$. This condition corresponds to Configuration A dealt with by Hall and Silkowski. ${ }^{5}$ Figure 2 shows the generalized force coefficient $C_{F 1}$ calculated with $\mu_{\max }=1$. This is highly similar to Fig. 5 of Hall and Silkowski's paper. $\left.{ }^{5}\right)$

In this case, the difference from the result for the isolated blade row is very small as seen in Fig. 2. But such a small effect of the neighboring blade row on the aerodynamic work is not alway the case, as will be discussed in Part II.

We have other evidence of code validation. As mentioned before, a comparison with the results of a CFD analysis for a three-dimensional rotor-stator blade row model was conducted by Hall et al. ${ }^{8)}$ and complete coincidence was demonstrated.

\section{Conclusions}

An analytical method to calculate unsteady aerodynamic force on the oscillating blades of contra-rotating annular cascades is developed as an extension of the unsteady lifting surface theory for a single rotating blade row.

A simple harmonic oscillation of blades results in unsteady blade loading of multiple frequency components because of aeroacoustic interaction between the blade rows in relative rotational motion. Simultaneous integral equations for all frequency components of the unsteady blade loadings of both blade rows are derived. The validity of the coded computation program is confirmed.

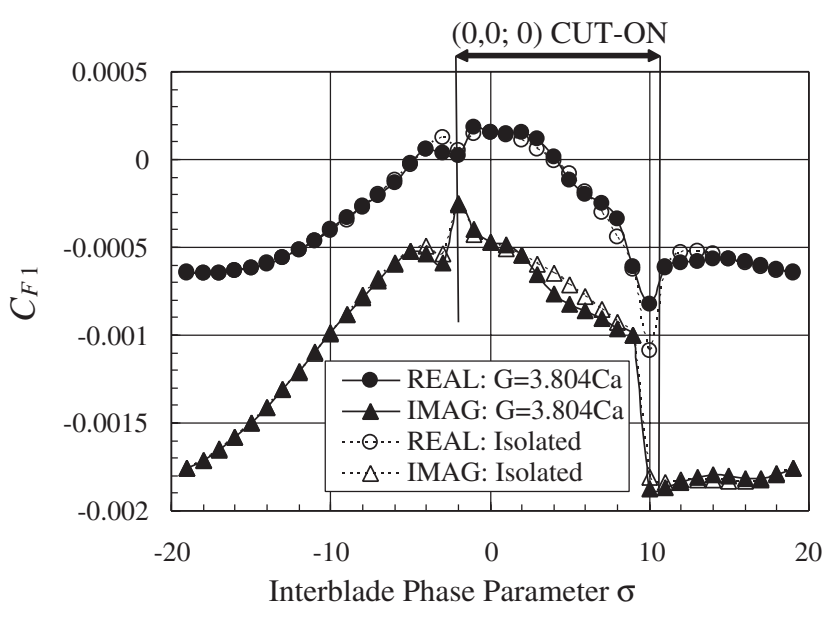

Fig. 2. Generalized force coefficient for Configuration A. ${ }^{5)}$ 


\section{References}

1) Tanida,Y.: Effect of Blade Row Interference on Cascade Flutter, Trans. Jpn. Soc. Aeronaut. Space Sci., 9 (1966), pp. 100-108.

2) Kobayashi, H., Tanaka, H. and Maruta, H.: Effect of Rotor-Stator Interaction on Cascade Flutter, 1st Report: Experimantal Study on Pure Bending Mode of Compressor Cascade, Trans. Jpn. Soc. Mech. Eng., 40 (1974), pp. 1615-1626.

3) Kobayashi, H., Tanaka, H. and Hanamura, Y.: Effect of Rotor-Stator Interaction on Cascade Flutter, 2nd Report: Theoretical Study on Pure Bending Mode, Trans. Jpn. Soc. Mech. Eng., 41 (1975), pp. 17701780.

4) Butenko, K. K. and Osipov, A. A.: Unsteady Subsonic Flow Past Two Relatively Moving Flat Cascades of Thin Weakly Loaded Oscillating Blades, Fluid Dynamics, Vol. 22, 0015-4628/88/2304-0620, Plenum Publishing Corporation, New York, 1989, pp. 620-625.

5) Hall, K. C. and Silkowski, P. D.: The Influence of Neighboring Blade Rows on the Unsteady Aerodynamic Response of Cascades, ASME J. Turbomachinery, 119 (1997), pp. 85-93.

6) Namba, M., Yamasaki, N. and Nishimura, S.: Unsteady Aerodynamic Force on Oscillating Blades of Counter-Rotating Annular Cascades, Proceedings of 9th International Symposium on Unsteady Aerodynamics, Aeroacoustics and Aeroelasticity of Turbomachines, Presses Universitaires de Grenoble, 2001, pp. 375-386.

7) Namba, M. and Nanba, K.: Unsteady Aerodynamic Work on Oscillating Annular Cascades in Counter Rotation: Combination of Supersonic and Subsonic Cascades, Unsteady Aerodynamics, Aeroacoustics and Aeroelasticity of Turbomachines, Hall, K.C., Kielb, R. and
Thomas, J.P., eds., Springer, 2006, pp. 177-188.

8) Hall, K. C., Ekici, K. and Voytovych, D. M.: Multistage Coupling for Unsteady Flows in Turbomachinery, Unsteady Aerodynamics, Aeroacoustics and Aeroelasticity of Turbomachines, Hall, K.C., Kielb, R. and Thomas, J.P., eds., Springer, 2006, pp. 217-229.

9) Namba, M. and Ishikawa, A.: Three-Dimensional Aerodynamic Characteristics of Oscillating Supersonic and Transonic Annular Cascades, ASME J. Eng. Power, 105 (1983), pp. 138-146.

10) Namba, M.: Three-Dimensional Flows, M.F. Platzer and F.O. Carta, eds., AGARD Manual on Aeroelasticity in Axial Flow Turbomachines, Vol. 1, Unsteady Turbomachinery Aerodynamics, AGARD-AG-298, Neuilly sur Seine, France, 1987.

11) Li, P., Toshimitsu, K. and Namba, M.: Double Linearization Theory for a Rotating Subsonic Annular Cascade of Oscillating Blades: (Part I, Mathematical Expressions of Disturbance Flow Field), Memoirs of the Faculty of Engineering, Kyushu University, 50 (1990), pp. 309340.

12) Li, P. and Namba, M.: Double Linearization Theory for a Rotating Supersonic Annular Cascade of Oscillating Blades, Memoirs of the Faculty of Engineering, Kyushu University, 50 (1990), pp. 161-185.

13) Schulten, J. B. H. M.: Sound Generated by Rotor Wakes Interacting with a Leaned Vane Stator, AIAA J., 20 (1982), pp. 1352-1358.

14) Namba, M. and Schulten, J. B. H. M.: Category 4 - Fan Stator with Harmonic Excitation by Rotor Wake: Numerical Results of Lifting Surface Theory, Third Computational Aeroacoustics (CAA) Workshop on Benchmark Problems, NASA/CP-2000-209790, 2000, pp. $73-85$ 